\title{
BMI open Trial-based cost-effectiveness analysis comparing surgical and endoscopic drainage in patients with obstructive chronic pancreatitis
}

To cite: Laramée $P$, Wonderling D, Cahen DL, et al. Trial-based costeffectiveness analysis comparing surgical and endoscopic drainage in patients with obstructive chronic pancreatitis. BMJ Open 2013;3:e003676. doi:10.1136/bmjopen-2013003676

- Prepublication history for this paper is available online To view these files please visit the journal online (http://dx.doi.org/10.1136/ bmjopen-2013-003676).

Received 28 July 2013 Accepted 28 August 2013

\footnotetext{
${ }^{1}$ National Clinical Guideline Centre, Royal College of Physicians, London, UK ${ }^{2}$ Department of Gastroenterology and Hepatology, Erasmus Medical Center, Rotterdam, The Netherlands

${ }^{3}$ Clinical Research Unit, Academic Medical Center, University of Amsterdam, Amsterdam, The Netherlands ${ }^{4}$ Department of Surgery, Academic Medical Center, University of Amsterdam, Amsterdam, The Netherlands ${ }^{5}$ UCL Institute for Liver and Digestive Health, University College London, London, UK
}

Correspondence to Dr Stephen P Pereira; Stephen.pereira@ucl.ac.uk

\section{ABSTRACT}

Objective: Published evidence indicates that surgical drainage of the pancreatic duct was more effective than endoscopic drainage for patients with chronic pancreatitis. This analysis assessed the costeffectiveness of surgical versus endoscopic drainage in obstructive chronic pancreatitis.

Design: This trial-based cost-utility analysis (ISRCTN04572410) was conducted from a UK National Health Service (NHS) perspective and during a 79-month time horizon. During the trial the details of the diagnostic and therapeutic procedures, and pancreatic insufficiency were collected. The resource use was varied in the sensitivity analysis based on a review of the literature. The health outcome was the Quality-Adjusted Life Year (QALY), generated using EQ-5D data collected during the trial. There were no pancreas-related deaths in the trial. All-cause mortality from the trial was incorporated into the QALY estimates in the sensitivity analysis.

Setting: Hospital.

Participants: Patients with obstructive chronic pancreatitis.

\section{Primary and secondary outcome measures:}

Costs, QALYs and cost-effectiveness.

Results: The result of the base-case analysis was that surgical drainage dominated endoscopic drainage, being both more effective and less costly. The sensitivity analysis varied mortality and resource use and showed that the surgical option remained dominant in all scenarios. The probability of costeffectiveness for surgical drainage was $100 \%$ for the base case and $82 \%$ in the assessed most conservative case scenario.

Conclusions: In obstructive chronic pancreatitis, surgical drainage is highly cost-effective compared with endoscopic drainage from a UK NHS perspective.

Chronic pancreatitis is a progressive inflammatory disorder, which can cause abdominal pain, various local complications and endocrine-exocrine pancreatic insufficiency.

\section{ARTICLE SUMMARY}

Strengths and limitations of this study

- The robustness of the results was assessed in the sensitivity analysis by varying relevant estimates using outcomes from reviews of the literature.

- All analyses were probabilistic; applying probability distributions to each model parameter and allowing estimation of the empirical distribution of the cost-effectiveness results.

- The limited randomised evidence on the topic led to develop this analysis based on a single trial with a relatively small sample size.

- The analysis did not include primary care costs associated with the follow-up of patients in the community. However, such costs are likely to be small compared with the cost of procedures and hospitalisation. This analysis was developed from a UK perspective and using collected data in the Netherlands. Caution is recommended before the results are extrapolated to other settings.

When chronic pancreatitis is associated with an obstructed pancreatic duct, a suitable therapy is ductal decompression, using an endoscopic or a surgical approach.

Published evidence having compared endoscopic and surgical procedures in patients with chronic pancreatitis and an obstructed pancreatic duct showed that surgical drainage of the pancreatic duct was more effective than endoscopic drainage in terms of pain relief and number of follow-up procedures. ${ }^{1-3}$ However, surgery is a more costly procedure than endoscopy and is believed to be associated with a higher risk of mortality. This trial-based economic analysis aimed to assess the cost-effectiveness of surgical drainage of the pancreatic duct compared with endoscopic drainage, for patients with chronic pancreatitis and an obstructed 
pancreatic duct, and combined resource use, cost, mortality and patient's quality-of-life data.

A cost-utility model was originally developed by the National Clinical Guideline Centre, Royal College of Physicians of London based on the 24-month aggregated resource use data from the Cahen trial ${ }^{1}$ This was conducted as part of the development process of the Clinical Guideline on Alcohol Use Disorders, which was commissioned and funded by the National Institute for Health and Care Excellence (NICE) ${ }^{4}$ This original analysis concluded that surgery was highly cost-effective compared with endoscopy and led to the recommendation by NICE that National Health Service (NHS) healthcare providers should 'Offer surgery, in preference to endoscopic therapy, to people with pain from large-duct (obstructive) chronic alcohol-related pancreatitis'. ${ }^{4}$ Second, when the long-term follow-up data from the Cahen trial (mean 79 months) became available, it was found that the cost per patient was $\$ 6006$ higher in the endoscopy group, but this difference was not statistically significant (95\% CI, $\$ 16188$ to $\$ 27786 ; p=0.29){ }^{3}$ At this time point, there were no longer differences in quality-of-life (SF-36) and health utility (EQ-5D based) scores. We present a trial-based cost-utility analysis, based on this long-term follow-up data.

The Cahen trial $^{1}{ }^{3}$ (the trial) included symptomatic patients with chronic pancreatitis and a distal obstruction of the pancreatic duct (without an inflammatory mass). Thirty-nine patients underwent randomisation: 19 to endoscopic transampullary drainage of the pancreatic duct and 20 to operative pancreaticojejunostomy. In the endoscopy group, following a sphincterotomy and dilation of the pancreatic duct stricture, a 10-French Amsterdam biliary stent was inserted and the stricture reassessed at 3 months. Persistent strictures were treated by repeated dilation and sequential insertion of multiple stents. Extracorporeal shock-wave lithotripsy was used if there were one or more intraductal stones more than $7 \mathrm{~mm}$ in diameter. In the surgical group, a pancreaticojejunostomy was performed in 18 patients by the method of Partington and Rochelle. The pancreatic duct was incised over the full length up to $2 \mathrm{~cm}$ from the ampulla. When retrieval of concretions from the head area required further opening of the duct towards the ampulla, a limited wedge resection of pancreatic tissue was performed. In one patient, a Whipple procedure was performed because of peripancreatic inflammation. In another patient, stone extraction required a Frey procedure. The baseline demographic and clinical characteristics of patients in the two treatment groups were similar, with the exception of ongoing alcohol abuse $(n=5$ in the surgery group; $n=0$ in the endoscopy group; $\mathrm{p}=0.05) .{ }^{1}$ One patient was lost to follow-up at 6 months after undergoing surgery and was excluded from the analysis. ${ }^{3}$ The results of the trial ${ }^{13}$ concluded that initial surgical drainage of the pancreatic duct is superior to endoscopic treatment in symptomatic patients with advanced chronic pancreatitis, not only based on short- term outcomes but also in the long term. These benefits apply for pain relief and the need for reintervention.

\section{METHOD \\ Overview}

This cost-effectiveness analysis was built from the trial data from randomisation to end of the long-term patient follow-up (mean of 79 months). ${ }^{1}{ }^{3}$ The trial was approved by the medical ethics committee of the Academic Medical Center, Amsterdam, controlled Trials number ISRCTN04572410.

The analysis was developed from an England and Wales NHS perspective using the NICE reference case. ${ }^{5}$ The measure of health outcome was the Quality-Adjusted Life Year (QALY). The time horizon used was the mean follow-up of 79 months of the trial. ${ }^{3}$ An annual discount rate of 3.5\% was applied to both costs and health outcomes incurred after 1 year, as is standard practice for health economic evaluations conducted for the NHS. ${ }^{5}$

\section{Utility scores}

In the trial, ${ }^{13}$ the EuroQol 5 dimensions questionnaire (EQ-5D $)^{6}$ was completed by patients (3-level EQ-5D). The EQ-5D is a generic health state preference measure. ${ }^{7}$ Data were collected for each arm at baseline, 6 weeks, 3, 6, 12, 18, 24 and 79 months. We used patientlevel EQ-5D data from the trial and generated utility scores for both arms at every follow-up point. The health state preference values (utilities) for EQ-5D profiles were based on time-trade-off valuations by members of the UK general public. ${ }^{7}$ Mean imputation was used to manage missing data. Because the baseline utility scores differed slightly between arms $(0.335$ vs 0.275 ; table 1$)$, the between-arm difference in utility score at each time point was adjusted for differences in baseline utility as proposed by Manca et al (2005), ${ }^{8}$ by applying ordinary least squares linear regression in SPSS 15.0 with baseline utility and trial arm as the only covariates. Because longterm EQ-5D data (post 24 months) were collected only at 79 months, and no difference between groups was demonstrated at 79 months (endoscopy $0.79 \pm 0.21$; surgery $0.82 \pm 0.26$; difference $-0.03,95 \%$ CI $(-0.20$ to $0.14), p=0.75)^{3}$, after 24 months we assumed no difference in utility score between the cohorts and applied a constant utility score of 0.79 (from the endoscopy group) to both groups. The QALYs in the endoscopy group were estimated by assuming a linear transition between the mean utilities at each time point (using the data from the endoscopy column of table 1). For the surgery group, the QALYs were also calculated assuming a linear transition (but at each time point the utility for surgery was the sum of the endoscopy utility and the increment of that time point from table 1).

\section{Mortality}

During the mean follow-up time of 79 months (SD, 24) of the study, ${ }^{3}$ three patients died in the endoscopy 


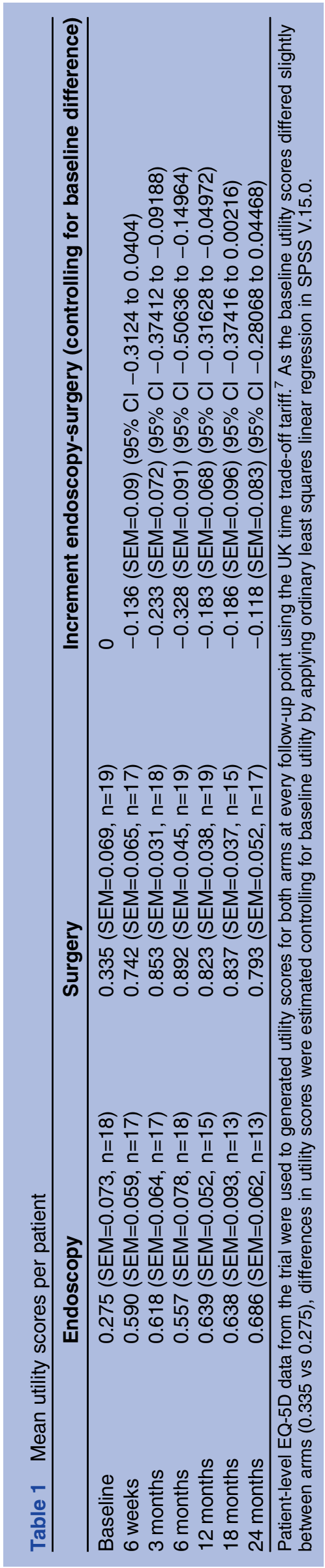

group and four in the surgery group. One early death was reported within 24 months: this endoscopically treated patient died of a perforated duodenal ulcer. After 24 months, another six patients died at a median time of 45 months (range, 27-59 months): two in the endoscopy group (pulmonary carcinoma; cardiovascular disease) and four in the surgery group (myocardial infarction; sepsis; neuroendocrine tumour; oropharyngeal carcinoma). As these deaths were unrelated to pancreatitis, these were not considered in the base case of this cost-effectiveness analysis but were included in the sensitivity analysis. In the sensitivity analysis, a utility of zero was applied from the death for each death in the trial.

\section{Resource use and costs}

The details of the use of diagnostic and therapeutic procedures, the treatment of pancreatic exocrine and endocrine insufficiency, and the time in hospital were collected during the trial. We combined this resource-use data with the most recent UK unit costs. ${ }^{9-11}$

Diagnostic procedures and therapeutic procedures (including the hospital stay) were costed using the 2010-2011 National Schedule of Reference Costs. ${ }^{9}$ Tables 2 and 3 present the diagnostic and the therapeutic procedures performed during the trial and their UK unit cost. ${ }^{13}$

Changes in pancreatic function (endocrine and exocrine) were assessed during the trial. Based on the 79-month results, ${ }^{3}$ and adjusting for baseline function, the proportion of patients for whom insufficiency persisted, resolved, developed and for whom sufficiency persisted were estimated for each trial arm. For exocrine insufficiency, treatment with pancreatic enzyme supplementations was costed for 79 months in patients whose insufficiency persisted (endoscopy $74 \%$; surgery $63 \%$ ), and for 39.5 months in patients whose insufficiency developed (endoscopy 26\%; surgery 26\%) or resolved (endoscopy $0 \%$; surgery $11 \%$ ). All patients were recorded as having exocrine insufficiency at some point. The treatment for exocrine insufficiency was assumed to be eight capsules a day of Creon 25000 current practice in England (daily cost of £2.26). ${ }^{10}$

For costing endocrine function, we used a yearly cost of $£ 939$ for a regimen of two injections per day of a biphasic insulin preparation. This is the lower yearly cost reported by the GINGER study economic evaluation assessing treatments for type 2 diabetes. ${ }^{12}$ This cost is still current according to the most recent MIMS, April $2013 .^{11}$ The treatment cost for endocrine function was calculated for 79 months in patients whose endocrine insufficiency persisted (endoscopy 26\%; surgery 26\%), and for 39.5 months in patients whose insufficiency developed (endoscopy 43\%; surgery 20\%). The remaining patients did not experience any endocrine insufficiency (endoscopy $31 \%$; surgery $54 \%$ ). 
Table 2 Diagnostic procedures

\begin{tabular}{|c|c|c|c|c|c|c|}
\hline \multirow[b]{2}{*}{$\begin{array}{l}\text { Procedure } \\
\text { (cost per procedure }^{9} \text { ) }\end{array}$} & \multicolumn{3}{|c|}{ Endoscopy group $(n=19)$} & \multicolumn{3}{|c|}{ Surgery group $(n=19)$} \\
\hline & $\begin{array}{l}\text { Number of } \\
\text { procedures }\end{array}$ & $\begin{array}{l}\text { Mean number } \\
\text { per patient }\end{array}$ & SD & $\begin{array}{l}\text { Number of } \\
\text { procedures }\end{array}$ & $\begin{array}{l}\text { Mean number } \\
\text { per patient }\end{array}$ & SD \\
\hline Abdominal CT scan (£168) & 13 & 0.68 & 0.95 & 26 & 1.37 & 1.46 \\
\hline $\begin{array}{l}\text { MR cholangiopancreatography } \\
\text { (£190) }\end{array}$ & 17 & 0.89 & 1.20 & 18 & 0.95 & 1.78 \\
\hline Abdominal ultrasound (£50) & 17 & 0.89 & 1.15 & 28 & 1.47 & 1.87 \\
\hline Endoscopic ultrasound (£591) & 5 & 0.26 & 0.45 & 1 & 0.05 & 0.23 \\
\hline Gastroscopy (£337) & 6 & 0.32 & 0.58 & 7 & 0.37 & 0.76 \\
\hline Colonoscopy (£638) & 3 & 0.16 & 0.50 & - & - & - \\
\hline Flexible sigmoidoscopy (£638) & 1 & 0.05 & 0.23 & - & - & - \\
\hline Radiography (£29) & 32 & 1.68 & 7.10 & 3 & 0.16 & 0.50 \\
\hline Missing data* (£157) & 2 & 0.11 & 0.32 & - & - & - \\
\hline All & 96 & 5.05 & 8.15 & 83 & 4.37 & 5.10 \\
\hline
\end{tabular}

\section{Sensitivity analysis}

Sensitivity analyses were performed to assess the robustness of the results. Two scenarios were tested. The first incorporated all-cause mortality from the long-term follow-up of the trial. ${ }^{3}$ The second was a more conservative case scenario, in which estimates for key parameters were used that were less favourable to surgical drainage.
In this scenario, parameters associated with the number of therapeutic procedures (the highest cost component) were altered from the base case: (1) conversion to surgery in the endoscopy group; (2) additional endoscopic drainage required in the endoscopy group and (3) additional surgical drainage required in the surgery group. In addition, the all-cause mortality from the trial

Table 3 Therapeutic procedures*

\begin{tabular}{|c|c|c|c|}
\hline Procedure (cost per procedure; mean hospital stay ${ }^{9}$ ) & $\begin{array}{l}\text { Number of } \\
\text { procedures }\end{array}$ & $\begin{array}{l}\text { Mean number } \\
\text { per patient }\end{array}$ & SD \\
\hline \multicolumn{4}{|l|}{ Endoscopy group $(n=19)$} \\
\hline $\begin{array}{l}\text { Extracorporeal shock-wave lithotripsy session (followed by an endoscopy } \\
\text { procedure) (£591; day case) }\end{array}$ & 27 & 1.42 & 1.22 \\
\hline $\begin{array}{l}\text { Extracorporeal shock-wave lithotripsy session (for stones, without subsequent } \\
\text { stenting) (£591; day case) }\end{array}$ & 6 & 0.32 & 0.67 \\
\hline Pancreaticojejunostomy ( $£ 6921 ; 6.6$ days) & 9 & 0.47 & 0.61 \\
\hline Endoscopic retrograde cholangiopancreatography ( $£ 1488 ; 2.2$ days) & 120 & 6.32 & 3.32 \\
\hline Endoscopic ultrasound-guided coeliac blockage (£902; day case) & 3 & 0.16 & 0.37 \\
\hline Cholecystectomy (£2844; 2.5 days) & 1 & 0.05 & 0.23 \\
\hline Hepaticojejunostomy and Frey procedure (£6921; 6.6 days) & 1 & 0.05 & 0.23 \\
\hline Frey procedure ( $£ 6921 ; 6.6$ days) & 1 & 0.05 & 0.23 \\
\hline Abscess drainage ( $£ 1488 ; 2.2$ days) & 1 & 0.05 & 0.23 \\
\hline Feeding tube insertion (nasojejunal tube) (£337; N/A) & 21 & 1.11 & 2.54 \\
\hline Transcutaneous electrical nerve stimulation (TENS) (£575; day case) & 1 & 0.05 & 0.23 \\
\hline Surgical block of splanchnic sympathetic nerve ( $£ 1701 ; 1.8$ days) & 1 & 0.05 & 0.23 \\
\hline Percutaneous gastrostomy ( $£ 919 ; 1.1$ days) & 1 & 0.05 & 0.23 \\
\hline Umbilical hernia procedure ( $£ 2004 ; 1.5$ days) & 1 & 0.05 & 0.23 \\
\hline Procedure for repair leakage ( $£ 6921 ; 6.6$ days) & 1 & 0.05 & 0.23 \\
\hline All & 195 & 10.26 & 5.67 \\
\hline \multicolumn{4}{|l|}{ Surgery group $(n=19)$} \\
\hline Frey procedure ( $£ 6921 ; 6.6$ days) & 1 & 0.05 & 0.23 \\
\hline Whipple procedure (£8469; 7.3 days) & 1 & 0.05 & 0.23 \\
\hline Pancreaticojejunostomy ( $£ 6921 ; 6.6$ days) & 17 & 0.89 & 0.32 \\
\hline Endoscopic retrograde cholangiopancreatography ( $£ 1488 ; 2.2$ days) & 8 & 0.42 & 1.61 \\
\hline Procedure for repair leakage (£6921; 6.6 days) & 1 & 0.05 & 0.23 \\
\hline Feeting tube insertion (nasojejunal tube) (£337; N/A) & 14 & 0.74 & 2.58 \\
\hline Distal pancreatic resection procedure (£6921; 6.6 days) & 1 & 0.05 & 0.23 \\
\hline Pain block procedure (£902; day case) & 4 & 0.21 & 0.63 \\
\hline All & 47 & 2.47 & 3.32 \\
\hline
\end{tabular}

${ }^{*}$ The cost of therapeutic procedures is including the cost for hospital stay. 
was also incorporated into this more conservative case scenario. ${ }^{3}$

\section{Conversion to surgery in the endoscopy group}

During the 79-month follow-up period of the trial, ${ }^{3} 47 \%$ $(9 / 19)$ of the patients in the endoscopy group converted to surgery as the endoscopic treatment was considered to have failed. Clinical studies assessing endoscopic drainage for treating patients with chronic pancreatitis were reviewed for the NICE Clinical Guideline on Alcohol Use Disorders ${ }^{4}$ for rates of conversion to surgery (databases were searched for the guideline up to 22 June 2009). It showed probabilities varying between $0 \%$ and $26 \%$ (overall $19 \%$ ). ${ }^{12}{ }^{13-20}$ This overall value of $19 \%$ was used in the sensitivity analysis.

\section{Additional endoscopic drainage required in the endoscopy group}

During the 79-month follow-up period of the trial, ${ }^{3} 68 \%$ $(13 / 19)$ of the patients in the endoscopy group required additional endoscopic drainage. Clinical studies assessing endoscopic drainage for treating patients with chronic pancreatitis were reviewed by the NICE Clinical Guideline on Alcohol Use Disorders ${ }^{4}$ for stent-related dysfunction/ complication rates (databases were searched for the guideline up to 22 June 2009). It showed probabilities varying between $3 \%$ and $55 \%$ (overall 15\%). ${ }^{15-19}{ }^{21}$ This overall value of $15 \%$ was used in the sensitivity analysis.

\section{Additional surgical drainage required in the surgery group}

During the 79-month follow-up period of the trial, ${ }^{3} 5 \%$ $(1 / 19)$ of the patients in the surgery group required additional surgical drainage. Clinical studies assessing surgery for treating patients with chronic pancreatitis were reviewed by the NICE Clinical Guideline on Alcohol Use Disorders $^{4}$ for rates of reoperation (databases were searched for the guideline up to 22 June 2009). After validation of this review, it showed reoperation rates varying between $2.6 \%$ and $17.5 \%$ (overall 8.8\%). ${ }^{1}{ }^{2}{ }^{22-25}$ The higher value of $17.5 \%$ was used in the sensitivity analysis.

\section{Mortality}

From a review of clinical studies for the NICE Clinical Guideline on Alcohol Use Disorders ${ }^{4}$ (databases were searched for the guideline up to 22 June 2009), the mortality related to surgical drainage was estimated to be $0.9 \% .^{122-29}$ During the mean follow-up time of 79 months of the trial, ${ }^{3}$ three patients died in the endoscopy group and four in the surgery group. These deaths were unrelated to pancreatitis, and thus not considered in the base case. All-cause mortality was, however, considered in the sensitivity analyses in which mortality risks of $15.8 \%(3 / 19)$ to the endoscopy group and $21.1 \%$ (4/19) to the surgical group were applied. This accounts for a difference of $5.3 \%$, a much higher estimate than those observed in the literature for mortality related to surgical drainage, allowing assessing a conservative case scenario against surgery.

\section{Statistical analysis}

This economic analysis, conducted in MS Excel 2010, presents all results probabilistically including sensitivity analyses. A probabilistic analysis, using Monte Carlo simulation, applies probability distributions to each model parameter, allowing estimation of the empirical distribution of the cost-effectiveness results. ${ }^{30} \mathrm{~A} \gamma$ distribution was applied to cost estimates (bounded at 0 ). The cost of therapeutic and diagnosis procedures, taken from the 2010 to 2011 National Schedule of Reference Costs, ${ }^{9}$ were varied using their IQR: the SE of each mean unit cost was estimated manually so that the 25th and 75th centiles of the $\gamma$ distribution to most closely fitted the published IQR of the unit cost. The costs for pancreatic insufficiency treatments were varied in a range of $\pm 20 \%$ using a uniform distribution. The distributions were applied to each unit cost before the unit costs were combined with the resource use frequency taken from the trial, and before discounting. For each item of resource use the frequency was given a $\beta$ distribution (bounded between 0 and 1). A $\beta$ distribution was also applied in the same context to the probability estimates for pancreatic function from the trial. A $\beta$ distribution was also applied to the mortality risks from the trial considered in the sensitivity analysis.

In addition to the adjustment for baseline imbalance from the trial applied to pancreatic function (endocrine and exocrine), baseline adjustment was applied to one other trial estimate as appropriate: the utility scores. As mentioned earlier, the between-arm difference in utility score at each time point was adjusted for differences in baseline utility by applying ordinary least squares linear regression. The resulting coefficient (increment for surgery) and its SE were then used as inputs in the probabilistic cost-effectiveness. More specifically, at each time point up to 24 months, a $\beta$ distribution was applied to the utilities of the endoscopy arm and a normal distribution to the increment for surgery estimated when adjusting for baseline differences (table 1; the distribution for each utility in the surgery arm was the sum of the utility in the endoscopy arm and the increment but was truncated at 1). Then, from 24 to 79 months, a $\beta$ distribution was applied to the constant same utility score considered for compared arms as explained earlier.

Results of the base-case and sensitivity analyses were recalculated 5000 times, with all model parameters set simultaneously, each selected at random from the respective parameter distribution. Results presented are the mean of the 5000 computed simulations. This approach was chosen to account for the uncertainty around the unit cost parameters as well as trial outcomes.

To estimate a two-sided $p$ value for the incremental cost we took the proportion of the 5000 simulations where costs were lower for endoscopy than for surgery and then multiplied by two. We applied the same approach to the QALYs gained. To estimate CIs, we took the 2.5th and 97.5th centiles from the 5000 simulations. 
Table 4 Base-case analysis results: mean costs and QALYs/patient*

\begin{tabular}{|c|c|c|c|}
\hline & $\begin{array}{l}\text { Endoscopy mean } \\
\text { (SD) }\end{array}$ & $\begin{array}{l}\text { Surgery mean } \\
\text { (SD) }\end{array}$ & Difference (endoscopy-surgery) \\
\hline Diagnostic procedures cost & $£ 831(208)$ & $£ 622(154)$ & $£ 210(221)(95 \% \mathrm{Cl}-186$ to 704$)(p=0.301)$ \\
\hline Therapeutic procedures cost & $£ 14797(3,393)$ & $£ 8853(2580)$ & $£ 5943(3334)(95 \% \mathrm{Cl} 86$ to 13290$)(p=0.047)$ \\
\hline $\begin{array}{l}\text { Pancreatic exocrine function } \\
\text { cost }\end{array}$ & $£ 4219(1936)$ & $£ 3956(1885)$ & $£ 264(779)(95 \% \mathrm{Cl}-1185$ to 1912$)(\mathrm{p}=0.701)$ \\
\hline $\begin{array}{l}\text { Pancreatic endocrine } \\
\text { function cost }\end{array}$ & $£ 2596$ (222) & $£ 1980(530)$ & $£ 616(503)(95 \% \mathrm{Cl}-420$ to 1546$)(p=0.225)$ \\
\hline Total cost & $£ 22443(3936)$ & $£ 15410$ (3262) & $£ 7033(3457)(95 \% \mathrm{Cl} 869$ to 14638$)(p=0.030)$ \\
\hline QALYs & $3.90(0.18)$ & $4.34(0.19)$ & $-0.44(0.09)(95 \% \mathrm{Cl}-0.61$ to -0.27$)(\mathrm{p}<0.001)$ \\
\hline
\end{tabular}

A limitation of our approach is that it does not capture the covariance between the utility at different time points or between resource use and utility, as each is considered independent. This is a limitation with regard to estimating the level of statistical significance but not with regard to our point estimates.

\section{RESULTS}

\section{Quality-adjusted life years}

We used the utility scores (endoscopy and increment) presented in table 1 to calculate QALYs for the 24-month trial duration, and applied, from 24 to 79 months to both groups the constant utility score of the endoscopy group at 79 months $(0.79 \pm 0.21) .{ }^{3}$ Considering the higher score at 24 months for the surgery group, this assumption after 24 months was conservative, that is to say biasing against surgery. When no difference in mortality was assumed, the QALY difference at 79 months was 0.44 in favour of surgery $(\mathrm{p}<0.001$; table 4$)$. When all-cause mortality from the trial was captured, the QALY difference still favoured surgery (difference of 0.22 , CI: -0.77 to 0.36 ), but the difference was no longer statistically significant (table 5).

\section{Resource use and cost}

Cost results are reported in 2011 pound sterling. Combining the frequency of each diagnostic and therapeutic procedure performed during the trial ${ }^{13}$ with UK unit costs from the 2010 to 2011 National Schedule of Reference Costs $^{9}$ (tables 2 and 3), we found a higher cost for endoscopy for both diagnostic and therapeutic procedures. However, the difference only reached statistical significance for the therapeutic procedures (£5943/ patient, 95\% CI: $£ 86$ to 13290 ; table 4 ). The costs of treating exocrine and endocrine insufficiency were higher for the endoscopy group but these differences did not reach statistical significance (table 4 ). The total cost for the base-case favoured surgery with a statistically significant difference of $£ 7033 /$ patient (95\% CI 869 to $14638)$.

In the sensitivity analysis, data from reviews of the literature were used to vary the cost of therapeutic procedures, so as to test a more conservative case scenario for surgical drainage. The surgery group showed a slightly lower cost for therapeutic procedures (£81, 95\% CI: $£ 5574$ to $£ 5780$ ). However, this difference was no longer statistically significant (table 5). The total cost for the sensitivity analysis favoured surgery, but this difference of $£ 1170$ did not reach statistical significance (95\% CI -4671 to 7066$)$.

\section{Cost-effectiveness}

The result of the base-case analysis was that surgical drainage of the pancreatic duct dominates endoscopic drainage (it was more effective and less costly-tables 4 and 6). The sensitivity analysis showed that the surgical option remains dominant (cost-saving and QALY increasing) in all scenarios, even under the assessed most conservative case scenario (table 6). In the sensitivity analysis, the total cost and QALY differences between surgery and endoscopy were not statistically significant (table 5). However, the probability that surgery is cost-

Table 5 Sensitivity analysis results: mean costs and QALYs/patient*

\begin{tabular}{lccr}
\hline & $\begin{array}{l}\text { Endoscopy mean } \\
\text { (SD) }\end{array}$ & $\begin{array}{l}\text { Surgery mean } \\
\text { (SD) }\end{array}$ & \multicolumn{1}{c}{ Difference (endoscopy-surgery) } \\
\hline Therapeutic procedures cost & $£ 9805(2328)$ & $£ 9725(2828)$ & $£ 81(2804)(95 \% \mathrm{Cl}-5574$ to 5780$)(\mathrm{p}=0.973)$ \\
Total cost & $£ 17451(3060)$ & $£ 16281(3462)$ & $£ 1170(2949)(95 \% \mathrm{Cl}-4671$ to 7066$)(\mathrm{p}=0.684)$ \\
QALYs (all-cause mortality) & $3.58(0.23)$ & $3.80(0.28)$ & $-0.22(0.28)(95 \% \mathrm{Cl}-0.77$ to 0.36$)(p=0.415)$ \\
\hline${ }^{*}$ Discounted at 3.5\%. & & & \\
QALY, Quality-Adjusted Life Year. & & &
\end{tabular}




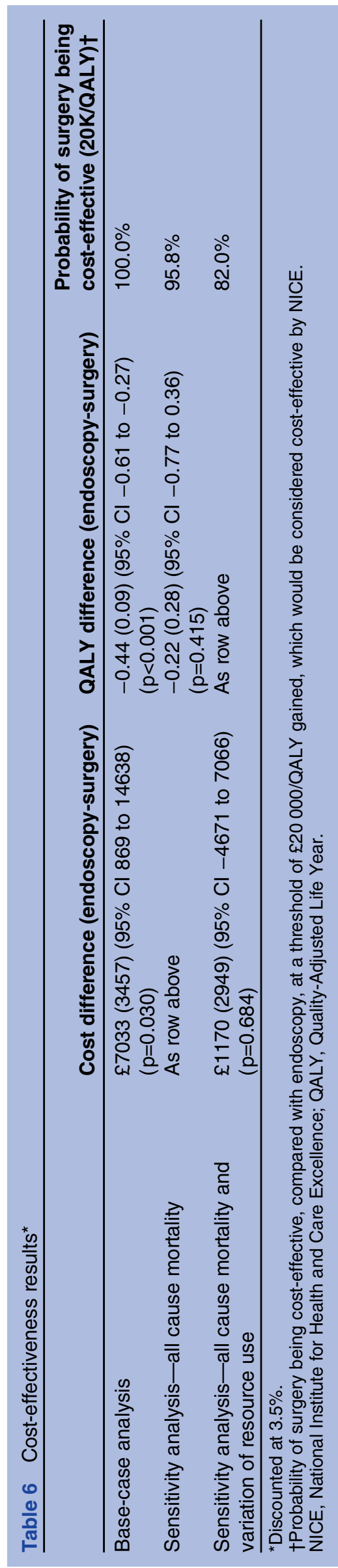

effective compared with endoscopy, assuming a threshold of $£ 20000 /$ QALY gained was $82 \%$ (100\% in the base case; table 6 and figure 1). The probability that surgery is cost-saving compared with endoscopy was $65.8 \%$ in the sensitivity analysis $(98.8 \%$ in the base case) .

\section{DISCUSSION}

On the basis of the 24-month aggregated results from the trial, ${ }^{1}$ an original cost-effectiveness model was developed to inform recommendations for the NICE Clinical Guideline on Alcohol Use Disorders. ${ }^{4}$ Based on the results from the model showing that surgery is costeffective compared with endoscopy, NICE recommended to 'Offer surgery, in preference to endoscopic therapy, to people with pain from large-duct (obstructive) chronic alcohol-related pancreatitis'.

The trial was extended to a long-term patient follow-up of a mean of 79 months, ${ }^{3}$ and the results led to conclude that, in patients with chronic pancreatitis and an obstructed pancreatic duct, initial surgical drainage of the pancreatic duct is superior to endoscopic treatment in symptomatic patients with advanced chronic pancreatitis, not only based on short-term outcomes but also in the long term. These benefits include greater pain relief and reduced need for reintervention. ${ }^{3}$ However, surgery is a more costly procedure than endoscopy, and therefore it has been unclear whether these benefits are greater enough to justify both the initial investment and the risks associated with surgery. We thus aimed to combine resource use, quality of life and mortality data from the trial, along with UK unit costs, to assess the cost-effectiveness of surgical drainage compared with endoscopic drainage.

This trial-based cost-effectiveness analysis was developed from a UK perspective and using data collected in the Netherlands. The trial results are transferable to the UK because of the reasonable similarity in terms of patient population, clinical practices and healthcare organisation. It is common practice in health economics to estimate the cost-effectiveness of an intervention from the perspective of one health system using the best available trial evidence conducted in a different country. However, even if it is accepted that the results of this cost-effectiveness analysis are sound from a UK perspective, a comparison of care pathways and unit costs should be made before the results of this study are extrapolated to other settings.

The current economic analysis considered the cost of diagnosis procedures, treatment of pancreatic function and therapeutic procedures with the associated hospital stay. The cost of the therapeutic procedures included the original treatment for pancreatitis, retreatment(s) and the treatment of complications. The analysis did not include primary care costs associated with the follow-up of patients in the community. However, such costs are likely to be small compared with the cost of procedures and hospitalisation, and therefore would not affect the conclusions. 


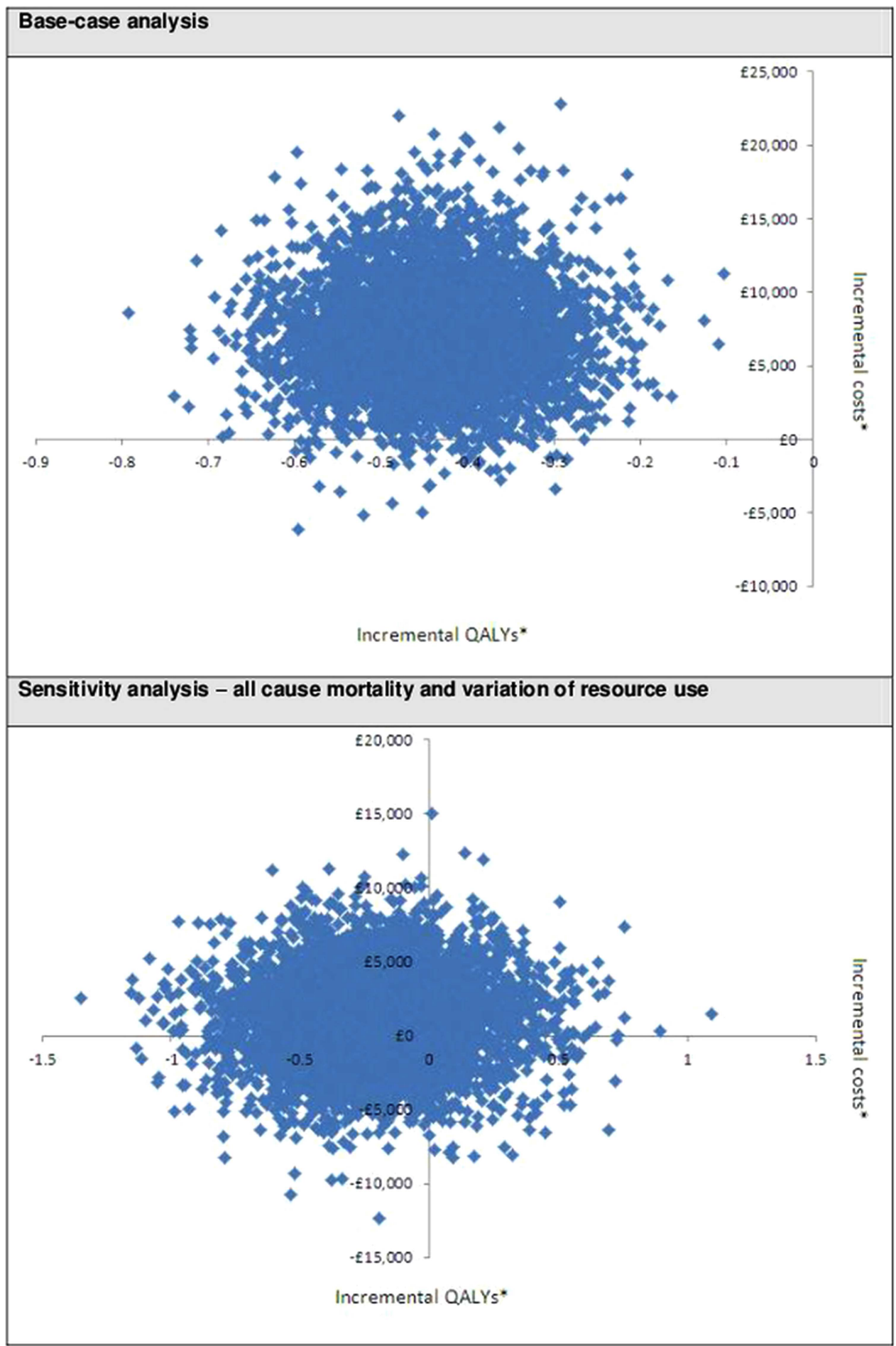

Figure 1 Cost-effectiveness scatter plots.

The limited randomised evidence on the topic led to this analysis being based on a single trial with a relatively small sample size. Nevertheless, the uncertainty around all estimates was accounted for by the probabilistic analysis, which allowed estimation of the empirical distribution of results and the statistical significance of the differences. Additionally, we conducted sensitivity analyses which confirmed the robustness of the conclusions that surgery is cost-saving and highly cost-effective compared with endoscopy, even under conservative assumptions.
Although the study size was relatively small, the probability that surgery is cost-effective was very high across the analyses. This was mainly due to the large improvements in quality of life at each follow-up point for the first 24-month period, as measured by the EQ-5D. The model showed that, in terms of QALYs, the large benefits from improved quality of life outweigh the QALYs lost due to increased risk of mortality, even under a conservative assumption: the difference in all-cause mortality of $5.3 \%$ applied in the sensitivity analysis is much 
higher than the reported mortality associated with surgical drainage $(0.9 \%){ }^{4}$

The baseline demographic and clinical characteristics of patients in the two treatment groups were similar, with the exception of ongoing alcohol abuse $(n=5$ in the surgery group; $n=0$ in the endoscopy group; $p=0.05$ ). We did not adjust for this when analysing the EQ-5D data nor did we estimate productivity losses, but if we had these are both likely to favour surgery.

Another trial (Dite 2003 ${ }^{2}$ ) comparing endoscopic and surgical procedures in patients with chronic pancreatitis and an obstructed pancreatic duct showed that, in terms of pain relief, surgical drainage of the pancreatic duct was more effective than endoscopic drainage. No quality-of-life assessment was undertaken in this trial ${ }^{2}$ and limited resource-use evidence was reported. Furthermore, extracorporeal shock-wave lithotripsy was not used in the Dite 2003 trial, ${ }^{2}$ making it less relevant to current practice than the trial we used in our analysis. ${ }^{3}$ Nevertheless, outcomes from Dite $2003^{2}$ were considered in the sensitivity analysis by varying the rate of conversion to surgery and the reoperation rate, which did not change the conclusion of the base-case costeffectiveness assessment.

A retrospective study ${ }^{31}$ from Japan based on medical records compared the resource use and medical cost associated with endoscopic drainage versus surgical drainage in patients with painful chronic calcified pancreatitis. A total of 68 patients were classified into endoscopy group $(n=34)$ or surgery group $(n=34)$. Patients receiving endoscopy were further divided into two subgroups: a short-period group-patients who could discontinue serial pancreatic stenting within 1 year $(n=19)$; and a long-period group-patients who needed pancreatic drainage by serial endoscopic stenting for more than 1 year $(n=15)$. This study concluded that patients in the long-period endoscopy group required significantly longer hospital stays, more frequent hospitalisations and had higher medical expenses than both the short-period endoscopic treatment group and the surgery group. No difference was found between the short-period endoscopy group and the surgery group. This study is more open to bias being a retrospective observational study, and the results from the analysis have been influenced by the choice of subgroups compared. The results, however, do suggest that it may be less costly to initiate treatment with surgery or to change approach before 1 year in the case of serial endoscopic stenting.

The total cost results in the current trial-based economic analysis showed for the base case that surgical drainage is less costly than endoscopy and this difference is statistically significant. These results were driven by a difference in the incidence of subsequent therapeutic procedures. In a previous comparative cost analysis of the long-term (79-month) results from the trial, ${ }^{3}$ it was shown that surgery was less costly but this difference did not reach statistical significance. The main reason that this study did not show statistical significance when the current study does is due to the inclusion of the cost of treating endocrine insufficiency in the current study.

In conclusion, surgical drainage of the pancreatic duct is highly cost-effective compared with endoscopic drainage for treating patients with chronic pancreatitis and an obstructed pancreatic duct in England and Wales. This conclusion was robust to sensitivity analysis. Also this analysis demonstrates that surgery is cost saving compared with endoscopy when considering all cost components related to patient care in chronic pancreatitis. This trial-based cost-effectiveness analysis lends further support to the NICE recommendation to 'Offer surgery, in preference to endoscopic therapy, to people with pain from large-duct (obstructive) chronic alcoholrelated pancreatitis'. However, the results should only be generalised to other healthcare systems with caution.

Contributors PL contributed to the conception and design of the research, acquisition of data, analysis and interpretation of the data, statistical analysis, cost-effectiveness modelling, drafting of the article, critical revision of the article for important intellectual content. DW contributed to the conception and design of the research, critical revision of the article for important intellectual content, supervision. DLC, DJG and MB contributed to the acquisition of data, drafting of the article, critical revision of the article for important intellectual content. MGD contributed to the acquisition of data, statistical analysis, drafting of the article, critical revision of the article for important intellectual content. SPP contributed to the conception and design of the research, analysis and interpretation of the data, drafting of the article, critical revision of the article for important intellectual content and supervision.

Funding This economic analysis was developed at the National Clinical Guideline Centre, Royal College of Physicians of London. The original version of this economic analysis, extended and updated in this article, was conducted as part of the development process of the Clinical Guideline on Alcohol Use Disorders, which was commissioned and funded by the National Institute for Health and Care Excellence (NICE). The clinical trial (1) on which we based this economic analysis was supported by an unrestricted grant from AstraZeneca, the Netherlands.

Competing interests None.

Ethics approval Medical ethics committee of the Academic Medical Center, Amsterdam, controlled Trials number ISRCTN04572410.

Provenance and peer review Not commissioned; externally peer reviewed.

Data sharing statement Unpublished specifications of this analysis are available from the corresponding author.

Open Access This is an Open Access article distributed in accordance with the Creative Commons Attribution Non Commercial (CC BY-NC 3.0) license, which permits others to distribute, remix, adapt, build upon this work noncommercially, and license their derivative works on different terms, provided the original work is properly cited and the use is non-commercial. See: http:// creativecommons.org/licenses/by-nc/3.0/

\section{REFERENCES}

1. Cahen DL, Gouma DJ, Nio Y, et al. Endoscopic versus surgical drainage of the pancreatic duct in chronic pancreatitis. $N$ Engl J Med 2007;356:676-84.

2. Dite $\mathrm{P}, \mathrm{Ruzicka} M$, Zboril V, et al. A prospective, randomized trial comparing endoscopic and surgical therapy for chronic pancreatitis. Endoscopy 2003;35:553-8.

3. Cahen DL, Gouma DJ, Laramee P, et al. Long-term outcomes of endoscopic vs surgical drainage of the pancreatic duct in patients with chronic pancreatitis. Gastroenterology 2011;141: 1690-5. 
4. National Clinical Guideline Centre. Alcohol use disorders: diagnosis and clinical management of alcohol-related physical complications. London: National Clinical Guideline Centre, 2010.

5. National Institute for Health and Clinical Excellence. The guidelines manual. London: NICE, 2009.

6. Kind P. The EuroQoL instrument: an index of health-related quality of life. In: Spilker B, ed. Quality of life and pharmacoeconomics in clinical trials. Philadelphia, PA: Lippincott-Raven, 1996:191-201.

7. Dolan P. Modeling valuations for EuroQol health states. Med Care 1997;35:1095-108.

8. Manca A, Hawkins N, Sculpher MJ. Estimating mean QALYs in trial-based cost-effectiveness analysis: the importance of controlling for baseline utility. Health Econ 2005;14:487-96.

9. Department of Health. NHS reference costs 2010-11. UK: Department of Health, 2011.

10. British Medical Association and Royal Pharmaceutical Society of Great Britain. British National Formulary. London, UK: BMJ Group and RPS Publishing, 2013.

11. MIMS. April 2013. London: Haymarket Media Group, 2013.

12. Vora JP, Punekar YS, Keech ML. A cost comparison of a BasalBolus Regimen (Glargine and Glulisine) with a premixed insulin regimen in type 2 diabetes patients. The GINGER Study. $\mathrm{Br} J$ Diabetes Vasc Dis 2011;11:314-18.

13. Rosch T, Daniel S, Scholz M, et al. Endoscopic treatment of chronic pancreatitis: a multicenter study of 1000 patients with long-term follow-up. Endoscopy 2002;34:765-71.

14. Binmoeller KF, Jue $P$, Seifert $H$, et al. Endoscopic pancreatic stent drainage in chronic pancreatitis and a dominant stricture: long-term results. Endoscopy 1995;27:638-44.

15. Renou C, Grandval P, Ville E, et al. Endoscopic treatment of the main pancreatic duct: correlations among morphology, manometry, and clinical follow-up. Int J Pancreatol 2000;27:143-9.

16. Farnbacher MJ, Schoen $C$, Rabenstein $T$, et al. Pancreatic duct stones in chronic pancreatitis: criteria for treatment intensity and success. Gastrointest Endosc 2002;56:501-6.

17. Eleftheriadis N, Dinu F, Delhaye M, et al. Long-term outcome after pancreatic stenting in severe chronic pancreatitis. Endoscopy 2005;37:223-30.

18. Dumonceau JM, Costamagna G, Tringali A, et al. Treatment for painful calcified chronic pancreatitis: extracorporeal shock wave lithotripsy versus endoscopic treatment: a randomised controlled trial. Gut 2007;56:545-52.
19. Smits ME, Badiga SM, Rauws EA, et al. Long-term results of pancreatic stents in chronic pancreatitis. Gastrointest Endosc 1995;42:461-7.

20. Cremer M, Deviere J, Delhaye M, et al. Stenting in severe chronic pancreatitis: results of medium-term follow-up in seventy-six patients. Endoscopy 1991;23:171-6.

21. Brand B, Kahl M, Sidhu S, et al. Prospective evaluation of morphology, function, and quality of life after extracorporeal shockwave lithotripsy and endoscopic treatment of chronic calcific pancreatitis. Am J Gastroenterol 2000;95:3428-38.

22. Lucas CE, McIntosh B, Paley D, et al. Surgical decompression of ductal obstruction in patients with chronic pancreatitis. Surgery 1999;126:790-7.

23. Schnelldorfer T, Adams DB. Outcome after lateral pancreaticojejunostomy in patients with chronic pancreatitis associated with pancreas divisum. Am Surg 2003;69: 1041-4.

24. Adams DB, Ford MC, Anderson MC. Outcome after lateral pancreaticojejunostomy for chronic pancreatitis. Ann Surg 1994;219:481-7.

25. Sielezneff I, Malouf A, Salle E, et al. Long term results of lateral pancreaticojejunostomy for chronic alcoholic pancreatitis. Eur J Surg 2000;166:58-64.

26. Kalady MF, Broome AH, Meyers WC, et al. Immediate and long-term outcomes after lateral pancreaticojejunostomy for chronic pancreatitis. Am Surg 2001;67:478-83.

27. Terrace JD, Paterson HM, Garden OJ, et al. Results of decompression surgery for pain in chronic pancreatitis. HPB 2007:9:308-11.

28. Madura JA, Canal DF, Lehman GA, et al. Wall stent-enhanced lateral pancreaticojejunostomy for small-duct pancreatitis. Arch Surg 2003;138:644-50.

29. Rios GA, Adams DB, Yeoh KG, et al. Outcome of lateral pancreaticojejunostomy in the management of chronic pancreatitis with nondilated pancreatic ducts. J Gastrointest Surg 1998;2: 223-9.

30. Doubilet $\mathrm{P}$, Begg $\mathrm{CB}$, Weinstein MC, et al. Probabilistic sensitivity analysis using Monte Carlo simulation. A practical approach. Med Decis Making 1985;5:157-77.

31. Hirota M, Asakura T, Kanno A, et al. Long-period pancreatic stenting for painful chronic calcified pancreatitis required higher medical costs and frequent hospitalizations compared with surgery. Pancreas 2011;40:946-50. 